\title{
Polyaniline Doped with Protonic Acids: Optical and Morphological Studies
}

\author{
Ravi V. Ingle, ${ }^{1}$ Shoaib F. Shaikh, ${ }^{2,}$ Pankaj K. Bhujbal, ${ }^{1}$ Habib M. Pathan ${ }^{1}$ and Vilas A. Tabhane ${ }^{1, *}$
}

\begin{abstract}
In this work, polyaniline (PANI) and its doped state with microfiber morphologies were easily synthesized by a chemical route under the oxidative polymerization, in which the polymerization was performed in an acidic medium (protonic acids). In situ polymerization of aniline reacted with the chloride and sulfate ions of hydrochloric and sulfuric acid, respectively. The microfiber-like morphology of undoped and $\mathrm{HCl}$ doped PANI was observerd. The X-Ray diffraction patterns represented that $\mathrm{H}_{2} \mathrm{SO}_{4}$ doped PANI was crystalline in nature. The effects of dopant acids on its optical properties were discussed. The strong interaction between chloride and sulfate ions with the ammonium group of PANI has been explained. The microfiber and granular morphology for $\mathrm{HCl}$ and $\mathrm{H}_{2} \mathrm{SO}_{4}$ doped PANI were observed. The redshifts were observed for the microfiber nature of doped PANI. These analytical results confirmed that the prepared PANI was in the doped state. Further, we found that the prepared PANI can be used for the fabrication of polymer solar cells.
\end{abstract}

Keywords: Polyaniline; Microfibers morphology; Doped PANI.

Received date: 24 May 2020; Accepted date: 8 June 2020.

Article type: Research article

\section{Introduction}

Polyaniline (PANI) is one of the most useful among the family of conjugated polymers as it is air and moisture stable in its conducting form (doping state) and insulating form (dedoped state). ${ }^{[1]}$ In the conjugated PANI polymers, the chemical bonding contains one unpaired $\pi$-electron having $\mathrm{sp}_{2} \mathrm{pz}$ configuration per carbon atom which leads to the electronic delocalization along the backbone of the polymer chain of PANI and provides the "highway" for the charge mobility to its doped structure. ${ }^{[2]}$ PANI is generally prepared by the chemical oxidative polymerization of aniline in the presence of strong oxidants such as ammonium persulfate (APS). ${ }^{[1]}$ Polymerization of aniline is an exothermic process, therefore the reaction is carried out at low temperatures $\left(0-10{ }^{\circ} \mathrm{C}\right)$ with one reactant (aniline) slowly added into APS under gentle stirring. ${ }^{[3]}$

Optical properties of parent PANI are different from those doped PANI due to the introduction of $-\mathrm{SO}_{3} \mathrm{H}$ and $\mathrm{Cl}^{-}$ groups along with the backbone structure of PANI. $-\mathrm{SO}_{3} \mathrm{H}$

\footnotetext{
${ }^{1}$ Advanced Physics Laboratory, Department of Physics, Savitribai Phule Pune University, Pune, India

${ }^{2}$ Department of Chemistry, College of Science, King Saud University, Riyadh 11451, Saudi Arabia

E-mail: yash.goaldriven@gmail.com(V.Tabhane)sshaikh1@ksu.edu.sa (S. Shaikh)
}

and $\mathrm{Cl}$ - ions create a more acidic medium, which is required for the polymerization of aniline. Recent theoretical calculation shows a strong steric interaction between the hydrogen atoms on adjacent rings of PANI because, after the doping of $\mathrm{H}_{2} \mathrm{SO}_{4}$, hydrogen atoms of PANI are replaced by $-\mathrm{SO}_{3} \mathrm{H}$ groups which lead to an increase of the steric hindrance. ${ }^{[4]}$ This steric hindrance limits the polymerization process of PANI. The size and amount of dopants play an important role in influencing the morphology of conducting polymers. The reported morphology of doped PANI (sulfonated porphyrin) can be changed from nanofibers to three- dimensional cauliflower structure. ${ }^{5]}$ PANI exhibits emeraldine salt structure when the PANI is obtained as a protonated form. ${ }^{[6]}$ Most of the PANI derivatives do not a well- defined fiber shape if it is prepared under the normal synthetic conditions. ${ }^{\text {[7] }}$

Previous synthesis of PANI (with its doped form) was carried out at low-temperature conditions (4 to $\left.0{ }^{\circ} \mathrm{C}\right) .{ }^{[3-5,7]}$ Here in this experiment we have synthesized PANI and its doped form at room temperature $\left(\approx 27^{\circ} \mathrm{C}\right)$. The objective of the present study is to investigate the changes observed in PANI after the doping with protonic acids $\left(\mathrm{HCl}\right.$ and $\left.\mathrm{H}_{2} \mathrm{SO}_{4}\right)$, and based on the reaction mechanism, the morphology of doped PANI has been studied. 


\section{Experimentat}

PANI was prepared by the direct addition of aniline into the APS solution. The molar ratio of aniline and APS (acts as an oxidizing agent) was $1: 1$ at room temperature $\left(25^{\circ} \mathrm{C}\right)$. PANI was doped with protonic acids viz. $\mathrm{HCl}$ and $\mathrm{H}_{2} \mathrm{SO}_{4}$ having different molarities $(0.5$ and $0.25 \mathrm{M})$. The solution was gently stirred (50-70 rpm) until it turned into a dark green and maintained overnight at room temperature. Then it was filtered using one filter paper. After filtration, the presipitate was dried. Accordingly, with increasing molarities, doped PANI was labeled as follows: "sample - b and c" for 0.25 and $0.5 \mathrm{M} \mathrm{H}_{2} \mathrm{SO}_{4}$ doped PANI, respectively whereas "sample - $\mathrm{d}$ and e" for 0.25 and $0.5 \mathrm{M} \mathrm{HCl}$ doped PANI, respectively; undoped PANI was labeled as "sample a".

\section{Characterization}

$\mathrm{X}$-ray diffraction (XRD) patterns for all dried powder samples of PANI were recorded on a Rigaku D/max-2400 $\mathrm{X}$-ray Diffractometer (Tokyo, Japan) using copper $\mathrm{K} \alpha$ radiation at $\lambda=0.154 \mathrm{~nm}$. Filtered liquid of all PANI samples was used for UV-visible spectra. Fourier transforms infrared (FTIR) spectra of the PANI were recorded on a Thermo Fischer FTIR spectrometer. FTIR spectra were collected from 4000 to $400 \mathrm{~cm}^{-1}$, with a $4 \mathrm{~cm}^{-1}$ resolution. The UVvisible absorption spectra were recorded on Perkin Elmer Lambda 950 spectrophotometer with $2 \mathrm{~nm}$ spectral resolution at room temperature in the range of 200-1000 nm. Scanning electron microscope (SEM) images of PANI were recorded using a JEOL JSM-6360A.

\section{Results and Discussion 4.1 FTIR study}

The presence of doping elements in the PANI was studied by FTIR as shown in Fig. 1. In the FTIR spectrum of PANI, Lesiak et al. ${ }^{[8]}$ ascribed $832 \mathrm{~cm}^{-1}$ bands to the out-of-plane bending vibration of $\mathrm{C}-\mathrm{H}$ on para disubstituted rings. FTIR spectrum of undoped and doped state of PANI shows a redshift of $37 \mathrm{~cm}^{-1}$ for the $795 \mathrm{~cm}^{-1}$ w. r. t. the $832 \mathrm{~cm}^{-1}$.

This redshift may be the indication of disturbed $\mathrm{C}-\mathrm{H}$ bending vibration in PANI. The band of $1030 \mathrm{~cm}^{-1}$ in all samples is correlated with sulfate ion from the APS (oxidant). ${ }^{[9]}$ The incorporation of protonic acids with PANI leads to the shifting of FTIR bands e.g. $1087 \mathrm{~cm}^{-1}$ is corresponding to $\mathrm{C}^{-\mathrm{N}^{+}}=\mathrm{C}$ radical cation stretching modes ${ }^{[10]}$ which is observed for sample - a, b, and c. The stretching mode $1146 \mathrm{~cm}^{-1}$ in $\mathrm{HCl}$ doped samples (d and e) has blue shifts around $60 \mathrm{~cm}^{-1}$. Seeger et al. ${ }^{[1]}$ stated that such blue shifts are due to the bond vibrations in phenyl rings of PANI, which are strongly perturbed by the charged polymers $\left(\mathrm{H}^{+} \mathrm{Cl}^{-}\right.$dopant ions). The perturbed mode, 1140$1146 \mathrm{~cm}^{-1}$ for sample - $\mathrm{c}$ and $\mathrm{e}$, is attributed to C-N stretching mode. ${ }^{[12]}$ However, in the sample-b and $c$, this stretching mode of $1146 \mathrm{~cm}^{-1}$ is absent which must be due to sulfate ions present in the chemical structure of the carbon chain of PANI. $1301 \mathrm{~cm}^{-1}$ mode is observed for both undoped and doped state of PANI, which is due to C-N delocalized polarons. ${ }^{[13]}$ This concludes that C-N delocalized polarons of PANI are not disturbed by the presence of protonic acids. FTIR spectra show $1486 \mathrm{~cm}^{-1}$ mode for the sample-a, $d$, and e which is assigned for $\mathrm{C}=\mathrm{C}$ of benzenoid rings, ${ }^{[14]}$ but the sample-b and $\mathrm{c}$ having redshifts of $66 \mathrm{~cm}^{-1}\left(1420 \mathrm{~cm}^{-1}\right)$ and $54 \mathrm{~cm}^{-1}\left(1432 \mathrm{~cm}^{-1}\right)$ respectively w.r.t. the $\mathrm{C}=\mathrm{C}$ benzenoid rings as shown in Fig. 1.

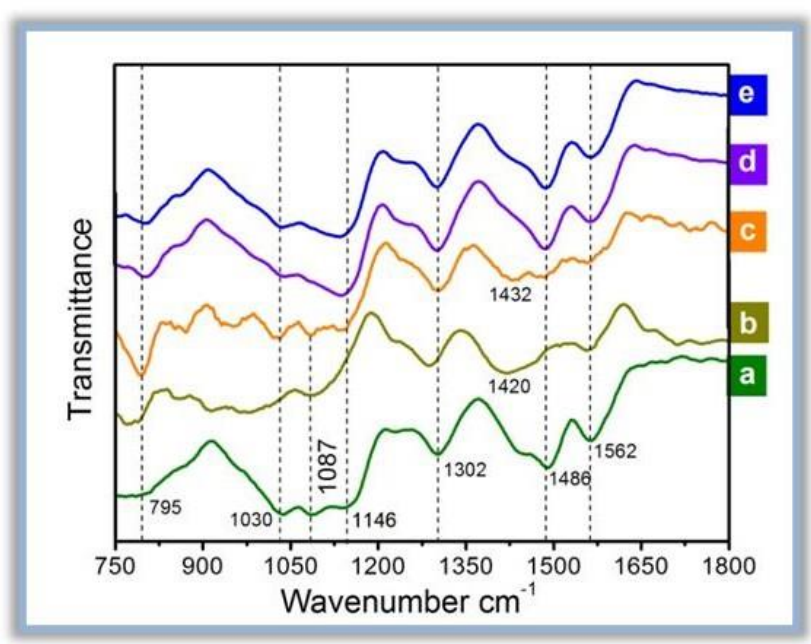

Fig. 1 FTIR spectra of undoped (sample-a) and doped PANI (sampleb to e). $\mathrm{H}_{2} \mathrm{SO}_{4}$ doped sample- $\mathrm{b}$ and c have some redshifts 1420 and $1432 \mathrm{~cm}^{-1}$ respectively towards longer wavenumber while there is a little influence in the $\mathrm{HCl}$ doped sample $\mathrm{d}$ and e over the vibration of wavenumber.

Redshifts indicate a strong interaction between $\mathrm{SO}^{2-}$ ians and the carbon chain of PANI. The $1562 \mathrm{~cm}^{-1}$ band is assigned for the $\mathrm{C}=\mathrm{C}$ bond in quinoid rings of PANI. ${ }^{[14]}$ This band was observed in both the undoped and doped state of PANI. Since sample -b and -c are doped by $\mathrm{H}_{2} \mathrm{SO}_{4}, \mathrm{SO}_{4}{ }^{2-}$ ions may have more influences on the quinoid rings of PANI. Due to the influence of this $\mathrm{SO}^{4-}$, sample-b, and -c might have the lowest transmittance inverse peak for this mode as compared to its undoped and $\mathrm{HCl}$ doped PANI. 1562, 1486, 1302,1146 , and $792 \mathrm{~cm}^{-1}$ are the characteristic transmittance inverse peaks for doped PANI. ${ }^{[15,16]}$

\subsection{UV-visible spectra}

The UV-vis spectra of PANI and its doped form are shown in Fig. 2(a) and 2(b). The broader peaks in UV vis spectra were observed at 378 and $530 \mathrm{~nm}$ for sample-b and $500 \mathrm{~nm}$ for sample-c as shown in Fig. 2(a). The broadening of these peaks revealed the transformation of PANI from its coiled to the extended coil form. ${ }^{[17]}$

The peak observed at $378 \mathrm{~nm}$ for sample- $\mathrm{b}$ is corresponding to $\pi-\pi^{*}$ transition in the benzenoid structure of PANI. ${ }^{[18,19]}$ Another peak for PANI at $530 \mathrm{~nm}$ has been assigned for the "exciton" transition ${ }^{[20-23]}$ from the highest 
occupied molecular orbital (HOMO) to the lowest unoccupied molecular orbital (LUMO). As reported earlier ${ }^{[4]}$ $\mathrm{H}_{2} \mathrm{SO}_{4}$ doped PANI has greater electron localization effects (induced by the $-\mathrm{SO}_{3} \mathrm{H}^{-}$groups) as compared to their parent PANI. The exciton transition peaks from 630 to $560 \mathrm{~nm}$ were reported by Stejska. ${ }^{[24]}$

In the present case, 500 and $530 \mathrm{~nm}$ absorption peaks were observed particularly for $0.5 \mathrm{M}$ (sample-c) and $0.25 \mathrm{M}$ (sample-d) $\mathrm{H}_{2} \mathrm{SO}_{4}$ doping concentrations, respectively. These excitons show the blue-shifts of 60 and $30 \mathrm{~nm}$ respectively w.r.t. the reported $560 \mathrm{~nm}$. Thus, these peaks must be associated with the excitons that occur within $\mathrm{H}_{2} \mathrm{SO}_{4}$ doped PANI. Hence, we can say that these blueshifted peaks are sensitive to the chemical structure of $\mathrm{H}_{2} \mathrm{SO}_{4}$ doped PANI. As reported, an absorption peak of 330 $\mathrm{nm}$ is the signature of the $\pi^{*}-\pi$ transition of benzenoid rings within the PANI structure. ${ }^{[25,26]}$

For sample- $\mathrm{b}$ and $-\mathrm{d}$, we have observed such absorption peaks at 375 and $378 \mathrm{~nm}$ having redshifts of 45 and $48 \mathrm{~nm}$ w.r.t. the reported $330 \mathrm{~nm}$. These redshifts confirmed the doping of PANI by protonic acids as shown in Fig. 2(b). As reported, the absorption peaks of $430 \mathrm{~nm}$ are attributed to the polaronic peak, indicating protonation of the backbone polymer chain of PANI. ${ }^{[6]} \mathrm{Wu}$ et al ${ }^{[27]}$ have studied the PANI nanofiber, they observed an absorption peak at $440 \mathrm{~nm}$ which is ascribed to the polaron- $\pi^{*}$ transition. Further, this peak indicates that PANI is in the doped state. ${ }^{[28]}$

In the present study, we have an absorption peak at 397 $\mathrm{nm}$ for sample-e ( $\mathrm{HCl}$ doped PANI), which has blue shifts of $43 \mathrm{~nm}$ w.r.t. the polaronic peak. This blue shift of $43 \mathrm{~nm}$ should be due to the microfiber behavior of PANI as we have observed in the present study. The absorption tail as shown in both parts of Fig. 2 is the extended "free- carrier tail" (NIR region: 800-1000 nm) of PANI which are the characteristics of charge carrier delocalization in the conducting polymers. ${ }^{[29]}$ Thus, due to $-\mathrm{SO}_{3} \mathrm{H}$ - group, the charges are more localized in sample-b and -c as compared to their pristine or undoped form (sample-a).

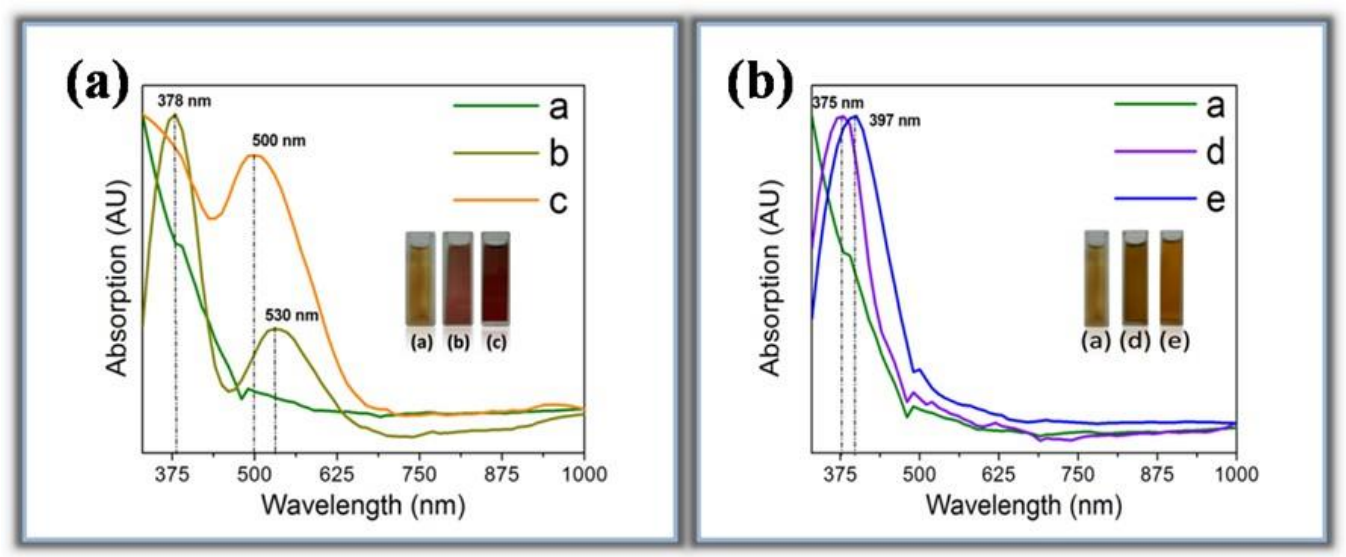

Fig. 2 UV-visible spectra: (a) Undoped and $\mathrm{H}_{2} \mathrm{SO}_{4}$ doped PANI (sample b and c). (b) Undoped and HCl doped PANI (sample d and e). Sample-b and $\mathrm{d}$ both have absorption peaks at around $375 \mathrm{~nm}$. The actual photograph of the undoped/doped PANI is shown in the inset of both Figures Undoped states have a yellow color and doped states of PANI have red and dark red color solutions.

\subsection{SEM analysis}

The mixed morphology of microfiber and granular coral type structures were observerd for the undoped PANI (sample -a) as shown in Fig. 3(a). Hence, the nucleation of sample - $\mathrm{a}$ is not only homogeneous but also heterogeneous. Upon $\mathrm{H}_{2} \mathrm{SO}_{4}$-doping (sample $-\mathrm{b}$ and -c), the microfiber structure of PANI almost disappears. Sample -b and -c revealed large chunks of doped PANI as shown in Fig. 3 (b) and 3(c). This granular morphology of $\mathrm{H}_{2} \mathrm{SO}_{4}$ doped PANI indicates a strong interaction between the $\mathrm{H}_{2} \mathrm{SO}_{4}$ and PANI during the polymerization. The interconnected network-like structures of $\mathrm{HCl}$ doped PANI microfibers are $1.6 \pm 0.5 \mu \mathrm{m}$ long. Jimenez et al. ${ }^{[30]}$ suggested that the origin of microfiber structures of $\mathrm{HCl}$ doped PANI is due to spontaneous self-nucleation during oxidative polymerization. Hence microfiber structure of sample -a, -d, and -e must have a homogenous polymerization. Since during the heterogeneous polymerization, the polymerized monomers of PANI grow on the previously formed PANI particles, these hetero nuclei particles act as nano-scale sticky contact linked to the particles together, leading to the agglomerated morphology of sample $-b$ and -c. ${ }^{[31,32]}$ Heterogeneous nucleation leads to the granular particulates morphology, which is observed for the sample-b and c. Hence $\mathrm{H}_{2} \mathrm{SO}_{4}$ couldn't help the monomers of PANI to form a microfiber structure as it has happened for $\mathrm{HCl}$ doped PANI as shown in Fig. 3(b) and 3(c). Diameters of undoped PANI microfibers are in the range of $0.12 \pm 0.02 \mu \mathrm{m}$ but after doping with $\mathrm{HCl}$, their diameters are expanded to 0.18 $\pm 0.02 \mu \mathrm{m}$ for the sample $\mathrm{d}$ and e as shown in Fig. 3(a), (d) and (f). The reasons behind the modification of the diameters of microfibers of sample $d$ and e are still unclear, but Zhao et $a l^{[13]}$ explained that this may be due to different sizes of the anion of acids and the dopant structure of polymers (hydrochloride emeraldine salt of PANI). Mattoso et $a{ }^{[33]}$ stated that the elongation process of PANI during the 
oxidative polymerization of aniline in an acidic medium involves reactive intermediate species $\left(\mathrm{H}^{+}\right.$and $\left.\mathrm{Cl}^{-}\right)$rearranged from the protonated aniline. The low concentration of protonated aniline is not beneficial to the rearrangement and elongation of reactive intermediate species because of low concentration of $\mathrm{H}^{+}$ions present in its acidic medium. ${ }^{[13]}$

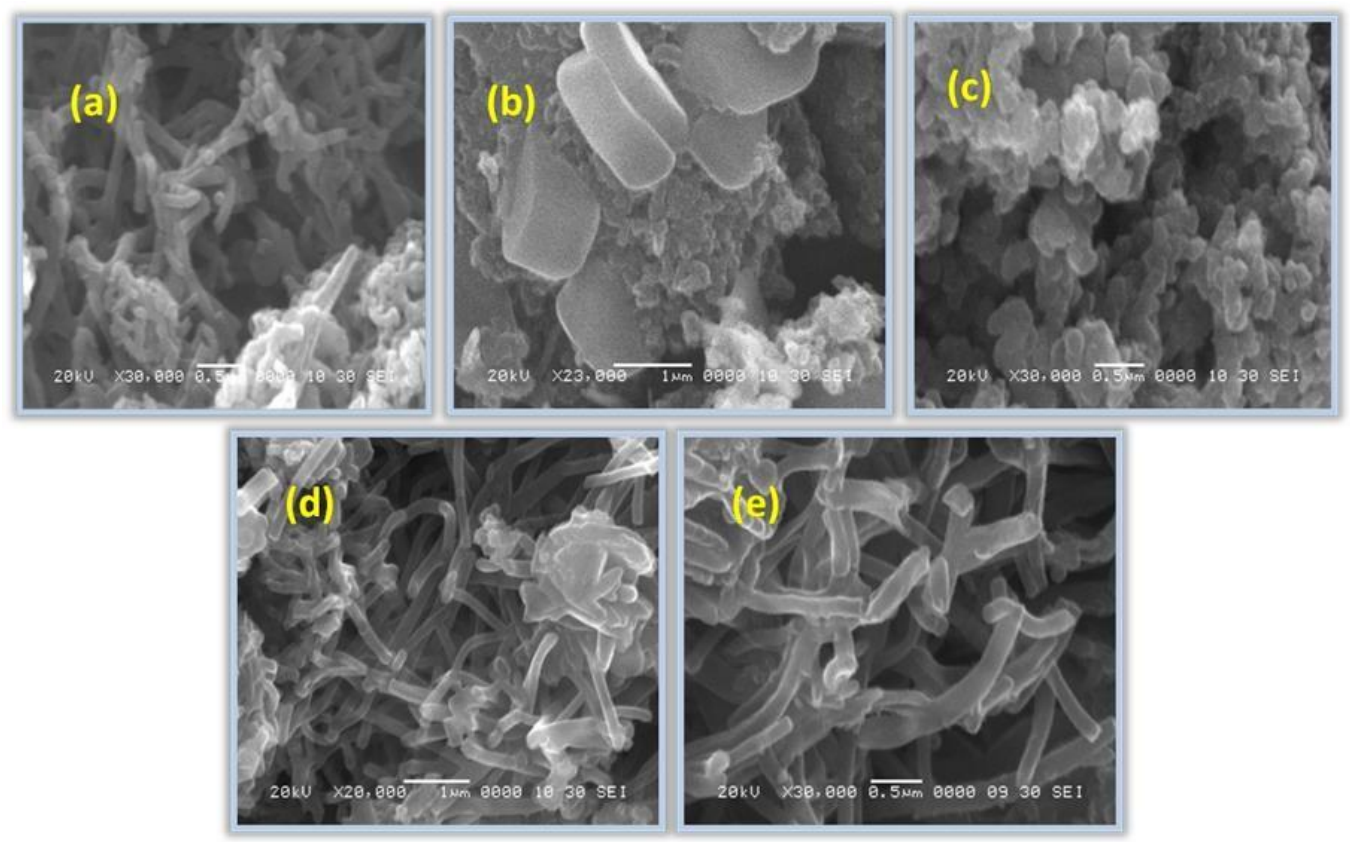

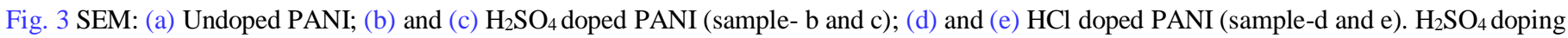
has forbidden the fiber growth of PANI while $\mathrm{HCl}$ doping is favorable for the growth of its fiber structure.

\subsection{Polymerization of undoped and doped PANI}

The present observation in the polymerization of PANI is fiber-like structures that were generated without adding any protonic acids. When the polymerization of PANI just turned into green, the collected sample contains almost welldispersed microfibers. If the oxidant (APS) is gradually added into the aniline with gentle stirring, the yielded microfibers become thicker and coarser and the final reaction solution contains mostly irregularly shaped microfibers of PANI. As the reaction moves on further, renucleation produces agglomerated microfibers of PANI. However, the study revealed that the homogeneous nucleation of PANI is responsible for the fiber structure of PANI. Zhang et al. ${ }^{[34]}$ found that the reaction temperature and the precursor ratio (1:1) of aniline/acid had an important influence on the morphology of the resulting PANI. As the polymerization of PANI is an exothermic reaction, lower temperature $\left(0-4{ }^{\circ} \mathrm{C}\right)$ is required for the formation of fiber morphology of PANI. However, in the present case, we have the microfibers of PANI at RT $\left(25^{\circ} \mathrm{C}\right)$. The formation of PANI was initiated by some nuclei (a small molecular aggregate), these nuclei can be formed by homogeneously in the parent phase of nucleation, or they can be heterogeneously grown on other species such as composite surface particles. ${ }^{[31,32]} \mathrm{We}$ propose that the kind of polymerization is controlled by the kind of dopant acids as well as the temperature because the polymerization in the present work changes due to dopant acids in PANI at RT. A similar result was observed by Stejskal et al. ${ }^{[35]}$ but they had explained the effect of acidity level of strong and weak acids on the PANI, in which organized nanotubular structures were obtained in a lower acidity at the lower temperature $(0$ $-4{ }^{\circ} \mathrm{C}$ ). In the present work, we have observed microfiber morphology of undoped and $\mathrm{HCl}$ doped PANI at the RT $\left(25{ }^{\circ} \mathrm{C}\right)$. Hence, reaction temperature plays an important role in getting the microfiber structure of PANI. The reported study revealed the fact that, as the aniline/acid ratio decreases $(<1: 1)$, nanostructured PANI was detected. ${ }^{[36,37]}$ The morphology of $\mathrm{H}_{2} \mathrm{SO}_{4}$ doped PANI has a granular structure that is often found in the literature. ${ }^{[38,39]}$ But none of the paper explains the exact reason for the granular morphology of $\mathrm{H}_{2} \mathrm{SO}_{4}$ doped PANI. However, the literature survey explained that the initial stage of polymerization of aniline oligomers is hydrophobic. These oligomers predetermine the directional growth of PANI. As the $\mathrm{pH}$ of the solution decreases, the hydrophobicity of the insitu synthesized PANI decreases and the corresponding PANI oligomer becomes soluble in the reaction medium, which results in oligomer aggregation of PANI structure. For the stronger acid, the smaller the value of dissociation constant $\left(\mathrm{pK}_{\mathrm{a}}\right)$, the higher is the dissociation of $\mathrm{H}^{+}$ions in the solution. ${ }^{[40]}$ The $\mathrm{pK}_{\mathrm{a}}$ values of $\mathrm{H}_{2} \mathrm{SO}_{4}$ and $\mathrm{HCl}$ are - 3 and $-6.3,{ }^{[41]}$ respectively. Thus, $\mathrm{HCl}$ is a stronger acid than $\mathrm{H}_{2} \mathrm{SO}_{4}$. Hence $\mathrm{HCl}$ doped PANI retains its acidity in the insitu polymerization of PANI, consequently its hydrophobicity is sustained for a long time which keeps the acidic environment for the polymerization of PANI even at RT. But as the reaction is exothermic and the temperature is 
not low, the diameters of fiber structure of the PANI are expanded and instead of nanofibers, microfiber morphology is observed for the undoped and $\mathrm{HCl}$ doped.

\subsection{XRD Analysis}

Dried undoped and doped PANI powder samples were examined by the XRD measurements as shown in Fig. 4. The sample - $\mathrm{b}$ and $-\mathrm{c}$ show diffraction peaks at $2 \theta=23.5$, 25.1 , and $30.3^{\circ}$ respectively. The peak of $25.1^{\circ}$ is the only feature of XRD patterns of undoped PANI (sample -a). The $\mathrm{X}$-ray reflection is at $25.1^{\circ}$ observed for all the samples of PANI which is ascribed for the regular spacing between phenyl rings of adjacent chains in a parallel orientation. ${ }^{[42]}$ The extra peaks at $23.3,26.3$, and $30.3^{\circ}$ for the sample $-\mathrm{b}$ and -c indicate that the doped PANI with $\mathrm{H}_{2} \mathrm{SO}_{4}$ is partially crystalline. In general, these extra peaks of sample - $b$ and $\mathrm{c}$ are influenced by the formation of a granular particle size of PANI as shown in Fig. 3(b) and 3(c). The X-ray peaks of PANI are the result of irregular arrangement of molecular chains whereas the regularity of the molecular chains does not show any X-ray peaks because, in this case, they are amorphous in nature. ${ }^{[43]}$ As the sample-a, $d$ and $e$ are microfibers, they have a regular arrangement of molecular chains of PANI polymers. Hence, there are not any noticeable X-ray peaks that are observed for sample $\mathrm{a},-\mathrm{d}$, and -e. However, the sample $-\mathrm{b}$ and $-\mathrm{c}$ have granular morphology of PANI and show the crystallinity behavior as to the amorphous nature of sample-a, d, and e. Hence there are X-ray peaks due to the randomly arranged aggregate polymer particles of PANI as shown in Fig. 4

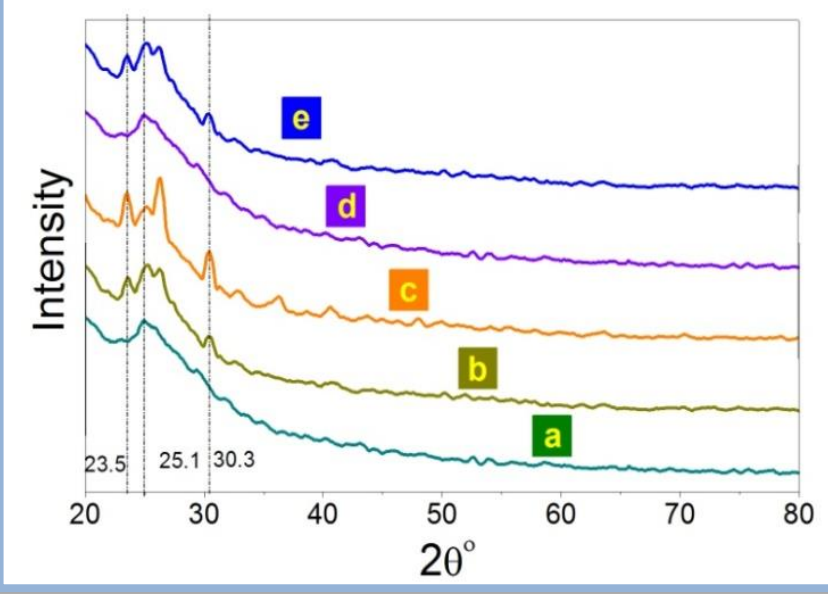

Fig. 4: XRD patterns of PANI prepared by the CBD method (a) undoped PANI, (b) $0.5 \mathrm{M} \mathrm{H}_{2} \mathrm{SO}_{4}$, (c) $0.25 \mathrm{M} \mathrm{H}_{2} \mathrm{SO}_{4}$, (d) $0.5 \mathrm{M} \mathrm{HCl}$ and (e) 0.25 $\mathrm{M} \mathrm{HCl}$ doped PANI. The extra peaks are obtained for sample- $\mathrm{b}$ and $\mathrm{c}$ which represents the crystallinity of aggregated particles of PANIproduced due to the influence of $\mathrm{H}_{2} \mathrm{SO}_{4}$ doping.

\section{Conclusion}

The optical and morphological study of undoped PANI was modified by the doping of protonic acids ( $\mathrm{HCl}$ and $\mathrm{H} 2 \mathrm{SO} 4)$. $\mathrm{XRD}$ analysis confirmed that the doped PANI with $\mathrm{H} 2 \mathrm{SO} 4$ is partially crystalline. Microfiber morphology was observed for $\mathrm{HCl}$ doped PANI, it is proved that $\mathrm{HCl}$ concentrations were favorable for the microfiber nucleation of PANI whereas $\mathrm{H} 2 \mathrm{SO} 4$ concentration forbided the microfiber nucleation of PANI. H2SO4 doped PANI produced large chunks of PANI and aggregation of PANI. Thus, it is easy to distinguish the $\mathrm{HCl}$ and $\mathrm{H} 2 \mathrm{SO} 4$ doped PANI. Room temperature and aniline/acid ratio (1:1) were responsible for the microfiber nature of $\mathrm{HCl}$ doped PANI. The redshift was observed for protonic acid doped PANI. The doping effect has a strong impact on the phenyl rings of PANI as compared to its undoped form. The optical spectrum shows that the absorption peak of $\mathrm{HCl}$ doped PANI was observed at $397 \mathrm{~nm}$, blue shifts of $43 \mathrm{~nm}$ was observed w.r.t. the polaronic peak at $440 \mathrm{~nm}$. This blue shift of $43 \mathrm{~nm}$ is a result of the microfiber behavior of $\mathrm{HCl}$ doped PANI whereas the redshifts in $\mathrm{H} 2 \mathrm{SO} 4$ in PANI represent that there are excitons trapped in the between $\pi$ - $\pi^{*}$ transitions of benzenoid rings. These excitons moved in a conduction band. Further, we found that the prepared PANI can be usedfor the fabrication of polymer solar cells.

\section{Acknowledgment}

HMP is thankful to the Departmental Research and Development program (DRDP) for partial financial support.

\section{Supporting information}

Not applicable

\section{Conflict of interest}

There are no conflicts to declare.

\section{References:}

[1] W. Huang, B. D. Humphrey and A. G. MacDiarmid, J. Chem. Soc. Faraday Trans., 1986, 82, 2385-2400, doi: 10.1039/ F19868202385.

[2] A. J. Heeger, J. Phys. Chem. B, 2001, 105, 8475-8491, doi: $10.1021 / \mathrm{jp} 011611 \mathrm{w}$.

[3] A. G. MacDiarmid, J. C. Chiang, A. F. Richter and A. J. Epstein, Synthetic Met., 1987, 18, 285-290, doi: 10.1016/0379-6779(87) 90893-9.

[4] J. Yue, Z. H. Wang, K. R. Cromack, A. J. Epstein and A. G. MacDiarmid, J. Am. Chem. Soc., 1991, 113, 2665-2671, doi: 10. 1021/ja00007a046.

[5] M. Khalid, M. A. Tumelero, I. S. Brandt, V. C. Zoldan, J. J. S. Acuña and A. A. Pasa, Ind. J. Mat. Sci., 2013, 2013, 718304, doi:10.1155/2013/718304

[6] J. P. Pouget, M. E. Jozefowicz, A. J. Epstein, X. Tang and A. G. MacDiarmid, Macromolecules, 1991, 24, 779-789, doi: 10.1021/ ma00003a022.

[7] J. X. Huang and R. B. Kaner, Chem. Commun., 2006, 4, 367-376, doi: 10.1039/B510956F.

[8] B. Lesiak, A. Jablonski, J. Zemek, M. Trchova and J. Stejskal, Langmuir, 2000, 16, 1415-1423, doi: 10.1021/la990699q.

[9] N. R. Chiou, L. J. Lee and A. J. Epstein, Chem. Mater., 2007, 19, 3589-3591, doi: 10.1021/cm070847v.

[10] R. V. Salvatierra, M. M. Oliveira and A. J. G. Zarbin, Chem. Mater., 
2010, 22, 5222-5234, doi: 10.1021/cm1012153.

[11] D. Seeger, W. Kowalchyk and C. Korzeniewski, Langmuir, 1990, 6, 1527-1534, doi: 10.1021/la00100a001.

[12] M. A. Habib and S. P. Maheswari, J. Electrochem. Soc., 1989, 136, 1050-1053, doi: 10.1149/1.2096782.

[13] M. Zhao, X. Wu and C. Cai, J. Phys. Chem. C, 2009, 113, $4987-$ 4996, doi: 10.1021/jp807621y.

[14] R. Sainz, W. R. Small, N. A. Young, C. Valles, A. M. Benito, W. K. Maser and M. I. H. Panhuis, Macromolecules, 2006, 39, 7324-7332, doi: 10.1021/ma061587q.

[15] W. Zheng, M. Angelopoulos, A. J. Epstein and A. G. MacDiarmid, Macromolecules, 1997, 30, 2953-2955, doi: 10.1021/ma9700136.

[16] F. Yan and G. Xue, J. Mater. Chem., 1999, 9, 3035-3039, doi: 10.1039/A905146E

[17] P. S. Khiew, N. M. Huang, S. Radiman and M. S. Ahmad, Mater. Lett., 2004, 58, 516-521, doi:10.1016/S0167-577X(03)00537-8.

[18] S. T. Selvan, T. Hayakawa, M. Nogami and M. Moeller, J. Phys. Chem. B, 1999, 103, 7441-7448, doi: 10.1021/jp991307r.

[19] A. Rahy, M. Sakrout, S. Manohar, S. J. Cho, J. Ferraris and D. J.Yang, Chem. Mater., 2008, 20, 4808-4814, doi: 10.1021/cm $703678 \mathrm{~m}$.

[20] W. B. Euler, Solid State Commun., 1986, 57, 857-859, doi: 10.1016/0038-1098(86)90166-3.

[21] C. B. Duke, A. Paton, E. M. Conwell, W. R. Salaneck and I. Lundstrom, J. Chem. Phys., 1987, 86, 3414-3423, doi: 10.1063/ 1.451998 .

[22] D. S. Boudreaux, R. R. Chance, J. F. Wolf, L. W. Shacklette, J. L. Brtdas, B. Themans, J. M. Andre and R. Silbey, J. Chem. Phys., 1986, 85, 4584-4590, doi: 10.1063/1.451779.

[23] S. Stafstram, J. L. BrCdas, A. J. Epstein, H. S. Woo, D. B. Tanner, W. S. Huang and A. G. MacDiarmid, Phys. Rev. Lett., 1987, 59, 1464-1467, doi: 10.1103/PhysRevLett.59.1464.

[24] J. Stejskal, M. Trchová, J. Prokeš and I. Sapurina, Chem. Mater., 2001, 13, 4083-4086, doi: 10.1021/cm011059n.

[25] Y. Guo and Y. Zhou, Eur. Polym. J., 2007, 43, 2292-2297, doi: 10.1016/j.eurpolymj.2007.01.020.

[26] T. Jana and A. K. Nandi, Langmuir, 2000, 16, 3141-3147, doi: 10.1021/la991151m.

[27] Y. Zhao, M. Arowo, W. Wu and J. Chen, Langmuir, 2015, 31, 51555163, doi: 10.1021/la504996c.

[28] Y. B. Zhao, W. Wu, J. F. Chen, H. K. Zou, L. L. Hu and G. W. Chu,
Ind. Eng. Chem. Res., 2012, 51, 3811-3818, doi: 10.1021/ ie202394c.

[29] A. G. MacDiarmid and A. J. Epstein, Synthetic Met., 1995, 69, 8592, doi: 10.1016/0379-6779(94)02374-8.

[30] P. Jimenez, P. Castell, R. Sainz, A. Anson, M. T. Martınez, A. M. Benito and W. K. Maser, J. Phys. Chem. B, 2010, 114, 1579 1585, doi: 10.1021/jp909093e.

[31] D. Li and R. B. Kaner, J. Am. Chem. Soc., 2006, 128, 968-975, doi: 10.1021/ja056609n

[32] D. Li and R. B. Kaner, J. Mater. Chem., 2007, 17, 2279-2282, doi: 10.1039/B700699C.

[33] L. H. C. Mattoso, A. G. MacDiamid and A. J. Epstein, Synthetic Met., 1994, 68, 1-11, doi: 10.1016/0379-6779(94)90140-6.

[34] Z. Zhang, Z. Wei and M. Wan, Macromolecules, 2002, 35, 59375942, doi: 10.1021/ma020199v.

[35] E. N. Konyushenko, J. Stejskal, I. Sedenkova, M. Trchova, I. Sapurina, M. Cieslar and J. Prokes, Polym. Int., 2006, 55, 31-39, doi: 10.1002/pi.1899.

[36] Y. Long, L. Zhang, Y. Ma, Z. Chen, N. Wang, Z. Zhang and M. Wan, Macromol. Rapid. Comm., 2003, 24, 938-942, doi: 10. 1002/marc.200300039.

[37] C. Cheng, J. Jiang, R. Tang and F. Xi, Synthetic Met., 2004, 145, 6165, doi: 10.1016/j.synthmet.2004.04.004.

[38] J. Stejskal, D. Hlavata, P. Holler, M. Trchov'a, J. Proke`s and I. Sapurina, Polym. Int., 2004, 53, 294-300, doi: 10.1002/pi.1406.

[39] J. Laska and J. Widlarz, Polymer, 2005, 46, 1485-1495, doi: 10.1016/j.polymer.2004.12.008.

[40] Aditi Sangal (2010) Advanced Organic Chemistry (2006) Krishna Publications Media (P) Ltd, Meerut, India

[41] K. Popov, H. Ronkkomaki and L. H. J. Lajunen, Pure Appl. Chem., 2006, 78, 663-675, doi: 10.1351/pac200678030663.

[42] R. V. Ingle, S. A. Arote, M. B. R. Prasad, V. S. Kadam, R. S. Mane, Inamuddin, M. Naushad, V. A. Tabhane and H. M. Pathan, High Perform. Polym., 2014, 26, 660-665, doi: 10.1177/0954008314539360.

[43] N. Gospodinova, P. Mokreva and L. Terlemezyan, Polymer, 1993, 34, 2438-2439, doi: 10.1016/0032-3861(93)90835-X.

Publisher's Note: Engineered Science Publisher remains neutral with regard to jurisdictional claims in published maps and institutional affiliations. 